\title{
Primeros datos sobre la colonización de la costa vasca por el gavión atlántico Larus marinus L., 1758.
}

\section{Colonisation of the Basque coast by the great black-backed gull Larus marinus L., 1758.}

Juan Arizaga ${ }^{1 *}$, Aitor Galarza $^{1}$

范

\section{Resumen}

Desde el primer caso de reproducción exitosa de la especie en la isla de Izaro (Bizkaia) en 2013, el gavión atlántico Larus marinus L., 1758 ha expandido su área de distribución a otras zonas de la costa vasca. El objetivo de este artículo es recopilar todas las citas disponibles sobre el proceso de colonización de esta especie que ha tenido lugar en las dos primeras décadas del presente siglo en la costa vasca. Esta colonización se inició a primeros de la década de 2010 y ha continuado en años posteriores. Durante el periodo 2012-2020, se localizaron 11 lugares en los que se detectó la presencia de gaviones emparejados en zonas aptas para la cría. Sólo tres llegaron a producir pollos (Izaro, Santa Clara y Txurruta). En 2020, la región albergaría un mínimo de tres parejas (en Santurtzi, Izaro y Santa Clara). Las parejas reproductoras se distribuyen por toda la costa, si bien de manera dispersa, en territorios que son defendidos, generalmente, dentro de colonias de gaviotas patiamarillas L. michahellis Naumann, 1840. El tamaño de puesta registrado en la costa vasca es de tres huevos (moda). La tasa de eclosión es de un 78,6\% $(n=14)$, pero el porcentaje de nidos con huevos que llega a producir pollos que vuelan es un $64,2 \%$. No se contabilizan en este cálculo las parejas en las que no llegó a haber puesta. Se confirma, en al menos 2 casos (Santa Clara y Txurruta), que el fracaso de las puestas se debe a molestias de origen humano, tanto en época de incubación como durante el momento de eclosión y cuidado de pollos. En otros casos, como Mollarri, es muy probable que las molestias causaran

\footnotetext{
1 Departamento de Ornitología, Sociedad de Ciencias Aranzadi Zorroagagaina 11, 20014 Donostia 
el abandono de los territorios antes de la puesta. Se anillaron pollos en Izaro, ininterrumpidamente a partir de 2013, y en Santa Clara en 2020.

Palabras clave: biogeografía, biología de la conservación, Laridae, molestias de origen humano, reproducción.

\begin{abstract}
Since its first breeding event on Izaro island (Bizkaia) in 2013, the distribution range of the great black-backed gull Larus marinus L., 1758 has expanded to other areas along the Basque coast. The aim of this paper is to compile all the breeding records of the great black-backed gull in order to document the colonisation process of this species along the aforementioned coastline. Colonisation started during the decade of 2010, continuing thereafter. During the period 2012-2020, we recorded 11 sites where the species was seen either breeding or paired in places adequate for breeding. Breeding was only detected at three sites (Izaro, Santa Clara y Txurruta). In 2020, the Basque coast would be the home for at least three pairs (in Santurtzi, Izaro and Santa Clara). The breeding pairs are found scattered along the whole coast in territories that are defended generally inside colonies of yellow-legged gull L. michahellis Naumann, 1840. The clutch size (mode) is 3 eggs, and the hatching rate is $78.6 \%(n=14)$, though the percentage of nests with eggs producing fledgling chicks is $64.2 \%$. It was confirmed at least in two instances (Santa Clara and Txurruta) that breeding failures were caused by disturbances of human origin, either during incubation or during the hatching and chick rearing period. In other cases, like Mollarri, it is reasonably likely that this type of disturbance caused an abandonment of the site even before the eggs were laid. Chicks were systematically ringed at Izaro from 2013, and in Santa Clara in 2020.
\end{abstract}

Key words: biogeography, conservation biology, Laridae, human disturbances, breeding.

\title{
Laburpena
}

Kaio handia, Larus marinus L. 1758, Izaro uhartean (Bizkaia) 2013an lehenengo aldiz arrakastaz ugaldu zenetik, euskal kostaldeko beste eremu batzuetara ere hedatu da. Artikulu honen helburua da biltzea euskal kostaldean mende honetako lehen bi hamarkadetan espezie honek izan duen kolonizazio prozesuaren aipamen guztiak. Kolonizazioa 2010eko hamarkadaren hasieran abiatu zen eta jarraitu egin du ondorengo urteetan. 2012-2020 aldian, ugaltzeko aproposak ziren 11 tokitan behatu dira kaio handien bikoteak. Horietatik hirutan baino ez dute lortu txitoak haztea (Izaro, Santa Klara eta Txurruta). 2020an kostako eremu horretan gutxienez hiru bikote egongo lirateke (Santurtzi, Izaro eta Santa Klara). Bikote ugaltzaileak kostaldean zehar hedatu dira, nahiko sakabanaturik bada ere, batez ere kaio hankahorien (Larus michahellis Naumann, 1840) kolonien barnean, eremu defendatuetan. Euskal kostaldean erregistratutako errunaldiaren tamaina hiru arrautzakoa da (moda). Eklosio-tasa \% 78,6 da $(n=14)$, baina arrautzak dituzten habietatik \% 64,2 dira hegan egiten duten txitoak sortzen dituztenak. Kalkulu honetarako ez dira barne hartzen 
errunaldi gabeko bikoteak. Gutxienez bi kasutan (Santa Klara eta Txurruta) baieztatu da ugaltzearen porrota gizakien jatorriko eragozpenen ondorioa izan dela, bai txitaldian eta bai zaintzeko garaian. Beste kasu batzuetan, Mollarrin esaterako, litekeena da eragozpenek eremuak abandonatzea eragin izana. Txitoak eraztundu dira Izaro uhartean etenik gabe 2013. urteaz geroztik eta Santa Klara uhartean 2020an.

Gako hitzak: biogeografía, kontserbazioaren biologia, Laridae, giza jatorriko eragozpenak, ugalketa.

\section{r. $2 x$}

\section{Introducción}

El gavión atlántico Larus marinus L., 1758 es un superdepredador marino distribuido a lo largo de las costas del norte del Atlántico, en Europa y Norteamérica, principalmente por encima del paralelo $45^{\circ} \mathrm{N}$ (Olsen \& Larson, 2004). Desde hace unos años, no obstante, ha expandido su área de distribución hacia nuevas áreas de cría, tanto en Europa (Bertolero et al., 2008, Dubois et al., 2008) como en Norteamérica (Ewins et al., 1992, Washburn et al., 2016). El primer caso de reproducción en la península ibérica se observó en 2005, en Os Farallóns (provincia de Lugo, Galicia) (Mouriño, 2005). Poco después, durante los censos que se llevaron a cabo a escala nacional en 2006-2007 se localizaron un total de 4 parejas, todas ellas en Galicia (Bertolero et al., 2008). A partir de entonces, el gavión ha colonizado el Cantábrico, criando por primera vez en Asturias en 2009 (Álvarez, 2009) y en la costa vasca en 2012 (aunque este año los huevos no llegaron a eclosionar; Galarza, 2013). Aunque en general la especie está en declive en Europa (Staneva \& Burfield, 2017), el gavión está experimentando un auge y expansión en el sur de su área de distribución (Bertolero et al., 2008, Issa \& Muller, 2015). Dada su reciente colonización, su estado de conservación no se ha evaluado en España hasta la fecha (Madroño et al., 2004),

Desde el primer caso de reproducción exitosa de la especie en la isla de Izaro (Bizkaia) en 2013 (Galarza, 2013), el gavión ha expandido su área de distribución a otras zonas de la costa vasca. El objetivo de este artículo es recopilar todas las citas disponibles sobre el proceso de colonización de esta especie en la costa vasca que ha tenido lugar en las dos primeras décadas del presente siglo. También se describen los principales parámetros reproductivos.

\section{Material y métodos}

El área tenida en cuenta en esta revisión abarca la línea de costa sudoccidental del Golfo de Vizcaya, desde el oeste de Bizkaia hasta el oeste del Departamento de Pyrénées Atlantiques (Fig. 1). Se cubre un periodo de 9 años, entre 2012 y 2020, esto es, 
todo el periodo con reproducción conocida de gaviones en la costa vasca desde la primera cita hasta la fecha.

Para documentar el proceso de colonización se han revisado todas las citas de reproducción (o intentos de reproducción), publicadas en trabajos científico-técnicos en revistas con revisión por pares así como fuentes de internet, tales como blogs o la plataforma Ornitho Euskadi (www.ornitho.eus). También se ha consultado a observadores que han llevado a cabo algún tipo de seguimiento de la reproducción de la especie durante el periodo de estudio. Además, el continuado seguimiento de una pareja en la isla de Izaro (Bermeo) ha proporcionado los primeros parámetros reproductivos de la especie en el área de estudio.

En la medida de lo posible, los pollos que han nacido durante todo este periodo fueron anillados (anilla metálica y anilla de PVC de lectura a distancia) con el fin de determinar su uso del territorio (una vez vuelan) y movimientos.

\section{Resultados}

Durante el periodo 2012-2020, se localizaron 11 lugares en los que se detectó la presencia de gaviones emparejados en zonas aptas para la cría. Sólo en tres de estas zonas se llegaron a producir pollos un mínimo de una vez en cada una de ellas (Fig. 1). A continuación se describe la información detallada recolectada en cada uno de los puntos con evidencias de reproducción (para más detalles ver, también, el Anexo 1). Para cada localidad (orden de oeste a este) se indica el topónimo y el municipio en paréntesis:

(1) Punta Lucero (Zierbana). En 2012 (Hidalgo et al., 2012) y 2013 (Galarza, 2013) se registra una pareja que defiende un territorio y construye nido, si bien no llega a realizar la puesta. No se vuelve a detectar la pareja en años posteriores.

(2) Puerto (Santurtzi). En 2020 se localiza una pareja que nidifica en el tejado de un pabellón (O. Carazo, G. Ocio, com. per.). Llega a realizar puesta, pero ésta fracasa por motivos que se desconocen.

(3) Billano (Gorliz). Observación de un ejemplar en 2015 y 2016 (marzo). En 2017 y 2018 (entre los meses de marzo a mayo) se observa una pareja que, no obstante, no construye nido. En 2020 sólo se cita un ejemplar (I. Zuberogoitia, com. per.).

(4) Aketx (Bermeo). Observación de una pareja que no construye nido, en 2016 (marzo y abril; I. Zuberogoitia, com. per.).

(5) Izaro (Bermeo). La reproducción en esta isla viene sucediendo ininterrumpidamente desde 2012. La puesta se perdió este primer año (Galarza, 2013), pero a partir de entonces la pareja ha producido un mínimo de 17 pollos (hasta 2020), de los que 16 han sido marcados para anillamiento (Tabla 1). 


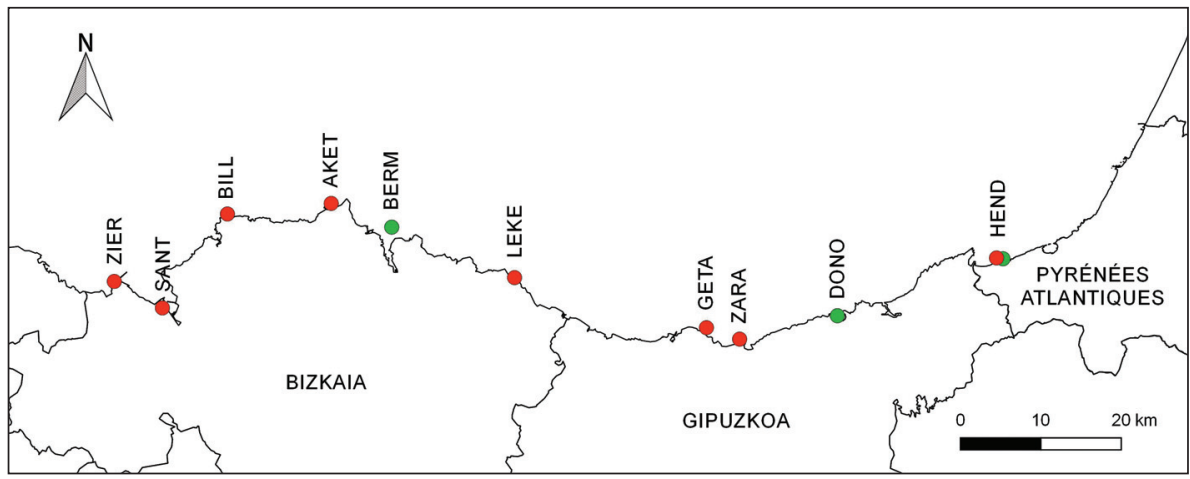

Fig. 1.- Localización de los puntos de cría de gavión en la costa vasca, durante el periodo 2012-2020. Círculos en rojo: localidades sin éxito; círculos en verde: localidades con éxito.

Fig. 1.- Breeding localities of great black-backed gulls along the Basque coast, during the period 20122020. Colour codes: red, localities without breeding success; green, localities with breeding success.

\begin{tabular}{|lccccccccc|}
\hline & 2012 & 2013 & 2014 & 2015 & 2016 & 2017 & 2018 & 2019 & 2020 \\
\hline Huevos & 2 & 3 & 3 & 3 & 3 & 3 & 3 & 3 & i? \\
\hline Pollos & 0 & 2 & 3 & 2 & 1 & 3 & 3 & 2 & 1 \\
\hline Eclosión & - & $21 / 05$ & $21 / 05$ & $25 / 05$ & $i ?$ & $15 / 05$ & $13 / 05$ & $05 / 05$ & $11 / 05$ \\
\hline
\end{tabular}

Tabla 1.- Número de huevos y pollos de la pareja de la isla de Izaro (Bermeo) en el periodo 2012-2020 y fechas aproximadas de eclosión. Se desconoce el número exacto de pollos de 2016, aunque se observó al menos un pollo volantón en la colonia.

Table 1.- Number of eggs and chicks produced by a pair breeding on Izaro island (Bermeo) during the period 2012-2020, and approximate hatching date. The exact number of chicks in 2016 remains unknown, although at least one chick was observed.

En 2012 se anilló la hembra y en 2014 el macho. En 2017 se observaron también en la colonia dos ejemplares sin anilla, aparentemente hembras. En 2019 el mismo macho anidó con una nueva hembra, que también fue anillada (Galarza, 2020). La Tabla 2 muestra algunos detalles sobre la morfología de estos tres adultos.

Como resultado del anillamiento han podido registrarse los movimientos de varios ejemplares: (1) en cuanto a pollos, se dispone de citas de 4 de ellos en su primer año de vida, de las que la más lejana se localiza a 242 km, en Gijón (Asturias), y el resto a menos de $15 \mathrm{~km}$ de Izaro. Dos de las recuperaciones son de ejemplares hallados muertos; (2) en cuanto a adultos, la observación más lejana se localiza a 24 km de Izaro, en Ondarroa (Bizkaia).

(6) San Nicolás-Garraitz (Lekeitio). En 2012 se registra una pareja que defiende un territorio pero no construye nido; sí lo hace en 2013, aunque no llega a realizar la puesta (J. Zubiaur en Galarza, 2013). La pareja se observa por última vez el 24/04/2013. Desde entonces no se ha vuelto a detectar en la zona en periodo de cría. 


\begin{tabular}{|lccccc|}
\hline Sexo & Anilla (PVC) & Peso $(\mathrm{g})$ & Pico $(\mathrm{mm})$ & Tarso $(\mathrm{mm})$ & Ala $(\mathrm{mm})$ \\
\hline Hembra & 3.BJR & 1490 & 22,8 & 84,4 & 478 \\
\hline Hembra & B:22 & 1600 & 23,7 & 92,0 & 474 \\
\hline Macho & B:05 & 1900 & 28,2 & 96,9 & 513 \\
\hline
\end{tabular}

Tabla 2.- Características morfológicas de tres gaviones adultos reproductores de la isla de Izaro (Bermeo). Medidas: Pico: altura máxima del pico; Tarso: longitud tarsal; Ala: longitud del ala -cuerda máxima-. Para cada ejemplar se indica, además, el código de anilla.

Table 2.- Morphological characteristics of three adult great black-backed gulls breeding on Izaro (Bermeo). Measurements: "Pico", bill depth; "Tarso", tarsus length; "Ala", wing length. We also show the ring colour code for each bird.

No hay evidencias que indiquen que el abandono del nido se produjera por molestias de origen humano (J. Zubiaur, com. per.).

(7) Getaria. En 2019 y 2020 se observa cortejo de una pareja que defiende un territorio en el cabo S. Antón, pero no construye nido (A. Aldalur, com. per.).

(8) Mollarri (Zarautz). Entre 2016 y 2018 se observa cortejo de una pareja que defiende territorio, pero no construye nido. El islote es frecuentado por bañistas que proceden desde la playa de Zarautz (A. Aldalur, com. per.).

(9) Santa Clara (Donostia). En 2016 se observa una pareja con comportamiento de cortejo que, sin embargo, no construye nido (J. Múgica, X. Saralegi -Itsas Enara Ornitologi Elkartea-; para más detalles ver ieoe.blogspot.com). Este comportamiento se repite en 2017 y 2018, y en 2019 se produce el primer evento reproductor (ver crónicas de toda la temporada de cría en el citado blog). Tras el fracaso de la primera puesta debido a molestias de origen humano, hubo una segunda que, lamentablemente, también fracasó (se constató la presencia reiterada de bañistas en la zona del nido cuando los huevos eclosionan en la última semana de junio en unos días de altas temperaturas; J. Arizaga, obs. per.; hecho también recogido en crónicas citadas anteriormente). En 2020, como consecuencia del confinamiento por el COVID-19, la ausencia de público permite que los gaviones pueden criar con éxito a pesar de ubicar el nido en una zona muy expuesta al paso de personas. La colocación de vallas para impedir el paso de público contribuye a evitar el fracaso. La pareja incuba tres huevos de los que llegan a eclosionar dos; los pollos procedentes de esos huevos fueron anillados. A mediados de julio un pollo, presumiblemente movido por varios visitantes de la isla que saltaron las vallas y trataron de establecerse en la zona del nido, fue recogido por observadores de Itsas Enara Ornitologi Elkartea y trasladado al centro de recuperación de fauna de la Diputación de Gipuzkoa (A. Lekuona, com. per.).

(10) Gemelas (Hendaia). En 2018 se registró una pareja que construyó un nido e hizo la puesta, aunque por causas desconocidas los huevos no eclosionaron (A. Alzaga, com. per.). Ni en 2019 ni en 2020 se observó la pareja durante el periodo de cría. 
(11) Txurruta (Hendaia). La reproducción de gavión se registra por primera vez en 2017 (Alzaga et al., 2018). De esta pareja, uno de cuyos componentes había sido anillado como pollo en 2012 en Niges (Charente-Maritime), $325 \mathrm{~km}$ al norte de Txurruta, llegó a verse un pollo. No obstante, éste nunca fue visto fuera de la isla. Presumiblemente no llegó a volar por las molestias generadas por una persona que acudió al islote reiteradamente en periodo de cría, aunque no se descartan otras hipótesis (Alzaga et al., 2018).

En la mayoría de los casos los gaviones establecieron su territorio en colonias de gaviotas patiamarillas L. michahellis Naumann, 1840, en general en islotes o islas. El nido a menudo se localiza en las zonas más prominentes de la colonia.

\section{Fenología de cría}

El establecimiento de territorio, cortejo, cópulas y construcción de nido sucede, mayoritariamente, entre marzo y abril (Tabla 3). La puesta tiene lugar habitualmente durante la segunda quincena de abril y el nacimiento de los pollos durante la segunda de mayo. Los pollos no volarían hasta al menos la primera mitad de julio (Tabla 3).

\begin{tabular}{|l|l|l|l|l|l|l|l|l|l|}
\hline & Mar1 & Mar2 & Abr1 & Abr2 & May1 & May2 & Jun1 & Jun2 & Jul1 \\
\hline Asentamiento, cortejo & & & & & & & & & \\
\hline Cópula & & & & & & & & & \\
\hline Construcción nido & & & & & & $*$ & & & \\
\hline Incubación & & & & & & & $*$ & & \\
\hline Nacimiento pollos & & & & & & & & $*$ & \\
\hline Pollos en nido & & & & & & & & & \\
\hline
\end{tabular}

Tabla 3.- Fenología de la reproducción de gavión atlántico en la costa vasca según la información documentada por varios observadores en diferentes publicaciones científico-técnicas y crónicas publicadas en internet. Intervalos temporales, en quincenas. Las celdas con asterisco hacen referencia a la fenología de segundas puestas.

Table 3.- Breeding phenology of the great black-backed gull on the Basque coast based on data provided by several authors, published in different journals, reports or internet blogs/pages. Time intervals, in fortnights. Cells with an asterisk refer to second broods.

\section{Parámetros reproductivos}

El tamaño de puesta registrado en la costa vasca es de tres huevos (moda). La tasa de eclosión es de un 78,6\% ( $n=14)$, pero el porcentaje de nidos con huevos que llega a producir pollos que vuelan es un 64,2\%. No se contabilizan en este cálculo las parejas en las que no llegó a haber puesta. Se confirma, en 2 casos al menos (Santa Clara y Txurruta), que el fracaso de las puestas se debe a molestias de origen humano, tanto 
en época de incubación como durante el momento de eclosión y cuidado de pollos. En otros casos, como Mollarri, es muy probable que éstas impidieran si quiera la puesta.

Los huevos miden de media 78,1 ( \pm SD: 2,4$) \times 52,1( \pm 1,4) \mathrm{mm}(n=21$; para ello se empleó un calibre digital).

En promedio, la productividad en Izaro es de 2,0 ( \pm SD: 1,1) pollos/pareja (para más detalles ver Tabla 1).

\section{Discusión}

La colonización de la costa vasca por el gavión atlántico se inició a primeros de la década de 2010 y ha continuado en años posteriores. En 2020, la región albergaría al menos un mínimo de tres parejas (en Santurtzi, Izaro y Santa Clara). Los gaviones crían en toda la costa, si bien de manera dispersa, en territorios que son defendidos, generalmente, dentro de colonias de gaviotas patiamarillas.

El hecho de que al menos una de las aves reproductoras se anillara como pollo en la costa francesa, al norte de Burdeos, indica que, posiblemente, la colonización de la costa vasca ha sido protagonizada, principalmente, por una expansión hacia el sur de la población atlántica de Francia. No obstante, no se debe descartar una posible expansión hacia el este de las parejas que crían en Galicia y el Cantábrico oriental. Asimismo, la edad de las aves con anilla permite sugerir que los ejemplares que han colonizado la costa vasca son, si no todos en parte, aves con poca experiencia, en su primer o primeros intentos de cría. Por otro lado, hasta la fecha (2020) no se ha detectado reclutamiento de los pollos que se han anillado en la zona.

En cuanto la morfología, las medidas que se han obtenido en los adultos que fueron anillados en Izaro se sitúan dentro de los rangos que se han descrito para la especie (Olsen \& Larson, 2004). La productividad en Izaro, con una media de 2 pollos/pareja, es alta, y comparable a la registrada en zonas donde la especie se reproduce a baja densidad (a densidades altas la media puede bajar a menos de 0,5 pollos/pareja) (Butler \& Trivelpiece, 1981).

Al margen de Izaro, la especie tiene un éxito reproductor bajo en toda la costa vasca. En parte este hecho podría estar asociado a la inexperiencia de los adultos así como a la nidificación aislada. En especies estrategas de la k (aves longevas con una inversión baja en la reproducción) es habitual que los primeros intentos de reproducción no sean exitosos (Newton, 1998). No obstante, también se observa que una fracción de las puestas fracasa por la existencia de molestias de origen humano. Aunque no se ha detectado hasta la fecha destrucción de puestas o pollos, la presencia de personas 
en el entorno del nido favorece el abandono de puestas y la muerte de pollos. Así, la presencia de público en la isla de Santa Clara, en Donostia, motivó el fracaso de la primera puesta de 2019, en fase de incubación, y de la puesta de reposición en el instante en que los huevos eclosionaban. Esto se produjo, además, a pesar de la valla y los carteles que se instalaron para proteger esta nidada. Un problema similar se observó en el islote de Txurruta en Hendaia (Alzaga et al., 2018). En la actualidad, únicamente tres de las localidades de cría conocidas (Billano, Izaro y Aketx) cuentan con un nivel de protección ambiental suficiente para garantizar un proceso reproductor exitoso. Por ello, para consolidar la reproducción de la especie en la costa vasca es crucial plantearse medidas eficaces de protección de los puntos de cría en época de reproducción, esto es, desde marzo hasta mediados de julio. En este contexto, la colaboración entre observadores particulares, instituciones y administraciones públicas puede ser clave. Por último, sería necesario incluir al gavión atlántico en el Catálogo Vasco de Especies Amenazadas, al tratarse de una especie con población muy pequeña que, además, está localizada en un área dispersa.

\section{Agradecimientos}

Agradecemos a los observadores que han suministrado datos inéditos sobre la especie a lo largo de todos estos años: Ó. Carazo y G. Ocio (Santurtzi), I. Zuberogoitia (Billano, Aketx), J. Zubiaur (Lekeitio), A. Aldalur (Zarautz y Getaria), J. Múgica y X. Saralegi (Donostia), A. Alzaga (Hendaia). A. Valiente colaboró durante la jornada de marcaje en la isla Santa Clara en 2020. A. Cama y un revisor anónimo proporcionaron algunos comentarios que contribuyeron a mejorar una primera versión del trabajo.

\section{Bibliografía}

Álvarez, D., 2009. Gavión atlántico Larus marinus: Noticiario Ornitológico. Ardeola 56, 358-359.

Alzaga, A., Herrero, A., Pagoaga, A., 2018. Première reproduction du Goéland marin Larus marinus dans les Pyrénées-Atlantiques. Le Casseur d'Os 18, 142-145.

Bertolero, A., Genovart, M., Martínez-Abraín, A., Molina, B., Mouriño, J., Oro, D., Tavecchia, G., 2008. Gaviotas cabecinegra, picofina, de Audouin y tridáctila, y gavión atlántico en España. Población en 2007 y método de censo. SEO/BirdLife, Madrid.

Butler, R.G., Trivelpiece, W., 1981. Nest spacing, reproductive success, and behavior of the Great Black-Backed Gull (Larus marinus). Auk 98, 99-107.

Dubois, P.J., Le Maréchal, P., Olioso, G., Yésou, P., 2008. Nouvel inventaire des oiseaux de France. Delachaux et Niestlé, Paris. 
Ewins, P.J., Blokpoel, H., Ludwig, J.P., 1992. Recent extensions of the breeding range of Great Black-backed Gulls (Larus marinus) in the Great Lakes of North America. Ontario Birds 10, 64-71.

Galarza, A., 2013. Primera reproducción del gavión atlántico Larus marinus L., 1758 en el País Vasco (Golfo de Vizcaya). Munibe, Cienc. nat. 61, 161-164.

Galarza, A., 2020. Gavión atlántico Larus marinus: Noticiario Ornitológico. Ardeola 67, 212.

Hidalgo, J., Olondo, M., García-Serna, I., 2012. Gavión atlántico Larus marinus: Noticiario Ornitológico. Ardeola 59, 439.

Issa, N., Muller, Y., 2015. Atlas des oiseaux de France métropolitaine. Nidification et présence hivernale. LPO/SEOF/MNHN, Paris.

Madroño, A., González, C., Atienza, J.C., 2004. Libro Rojo de las Aves de España. DGBSEO/BirdLife, Madrid.

Mouriño, J., 2005. Primeros datos sobre nidificación de Gavión Atlántico (Larus marinus) en la Península Ibérica. VI Congreso Galego de Ornitología-V Jornadas Ornitológicas Cantábricas, Viveiro.

Newton, I., 1998. Population limitation in birds. Academic Press, London.

Olsen, K.M., Larson, H., 2004. Gulls of Europe, Asia and North America. Christopher Helm, London.

Staneva, A., Burfield, I., 2017. European birds of conservation concern: populations, trends and national responsibilities. BirdLife International.

Washburn, B.E., Elbin, S.B., Davis, C., 2016. Historical and current population trends of Herring Gulls (Larus argentatus) and Great Black-Backed Gulls (Larus marinus) in the New York Bight, USA. Waterbirds 39, 74-86.

Fecha de recepcion/Date of reception: 17/07/2020

Fecha de aceptacion/Date of acceptance: 28/08/2020

Editor Asociado/Associate editor: Iván de la Hera 
Anexo 1. Resumen de las evidencias que describen el proceso de colonización de gavión en la costa vasca. Código de localidades: ZIER, Zierbana; SANT, Santurtzi; BILL, Billano; AKET, Peñón de Aketx; BERM, Bermeo (Izaro); LEKE, Lekeitio (S. Nicolás); GETA, Getaria (S. Antón); ZARA, islote de Mollarri, Zarauz; DONO, Donostia (Santa Clara), HEND (Hendaia; 2 puntos de cría próximos, la isla de Txurruta y las Gemelas).

Annex 1. Compiled breeding evidence events describing the great black-backed gull colonisation process on the Basque coast. Locality code: ZIER, Zierbana; SANT, Santurtzi; BILL, Billano; AKET, Peñón de Aketx; BERM, Bermeo (Izaro); LEKE, Lekeitio (S. Nicolás); GETA, Getaria (S. Antón); ZARA, Mollarri, Zarauz; DONO, Donostia (Santa Clara), HEND (Hendaia; 2 nearby breeding places, Txurruta island, and the Gemelas).

\begin{tabular}{|c|c|c|c|c|c|c|c|c|c|c|}
\hline & ZIER & SANT & BILL & AKET & BERM & GETA & ZARA & LEKE & DONO & HEND \\
\hline 2012 & $\mathrm{~N}$ & & & & $\mathrm{H}$ & & & $\mathrm{T}$ & & \\
\hline 2013 & $N$ & & & & $P^{\prime}$ & & & $\mathrm{N}$ & & \\
\hline 2014 & 0 & & & & $P^{\prime}$ & & & 0 & & \\
\hline 2015 & 0 & & & & $P^{\prime}$ & & & 0 & & \\
\hline 2016 & 0 & & & $\mathrm{~T}$ & $P^{\prime}$ & & $\mathrm{T}$ & 0 & $\mathrm{~T}$ & \\
\hline 2017 & 0 & & $\mathrm{~T}$ & & $P^{\prime}$ & & & 0 & $\mathrm{~T}$ & $P$ \\
\hline 2018 & 0 & & $\mathrm{~T}$ & & $P^{\prime}$ & & $\mathrm{T}$ & 0 & T & $\mathrm{H}$ \\
\hline 2019 & 0 & & 0 & & $P^{\prime}$ & T & 0 & 0 & P & 0 \\
\hline 2020 & 0 & $\mathrm{H}$ & 0 & 0 & $P^{\prime}$ & T & 0 & 0 & $P^{\prime}$ & 0 \\
\hline
\end{tabular}

T, territorio, parada nupcial y cortejo, pero no llega a haber construcción de nido. N, nido construido; $\mathrm{H}$, huevos (no se detectan pollos); $\mathrm{P}$, pollos (no sobreviven o no se confirma que lleguen a volar); $\mathrm{P}^{\prime}$, pollos volantones; 0 , confirmada ausencia de pareja reproductora en la zona.

T, territory, courtship display and mating, but no nest is built. $N$, nest built; $H$, eggs (no chicks are detected); $\mathrm{P}$, chicks (these do not survive or fledging not confirmed); $\mathrm{P}^{\prime}$, fledglings; 0 , confirmed absence of reproductive pair in the area. 